The tool we propose could be a valid support in accurately assessing the joint and indirectly skin involvement of sclerodactyly in this type of patient, especially in the context of a clinical trial to evaluate the efficacy of a treatment.

Further studies are needed to compare with other methods to assess hand disability in SSc such as the use of HAMIS (Hand Mobility in Scleroderma) test. REFERENCES:

[1] Sandqvist G et al. J Rheumatol. 2016 Jul;43(7):1356-62.

[2] https://www.arduino.cc.

Disclosure of Interests: None declared

DOI: 10.1136/annrheumdis-2021-eular.1826

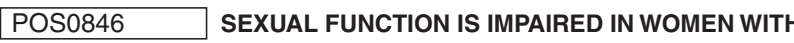 SYSTEMIC SCLEROSIS COMPARED TO HEALTHY CONTROLS}

B. Heřmánková ${ }^{1,2}$, M. Špiritović ${ }^{1,2}$, S. Oreska ${ }^{2,3}$, H. Štorkánováé, ${ }^{2,3}$, H. Smucrova ${ }^{2}$, K. Pavelka ${ }^{2,3}$, J. Vencovský ${ }^{2,3}$, L. Šenoltt ${ }^{2,3}$, R. Bečváŕ ${ }^{2,3}$, M. Tomčík ${ }^{2,3}$.

${ }^{1}$ Faculty of Physical Education and Sport, Charles University, Department of Physiotherapy, Prague, Czech Republic; ${ }^{2}$ Institute of Rheumatology, Department of Rheumatology, Prague, Czech Republic; ${ }^{3}$ First Faculty of Medicine, Charles University, Department of Rheumatology, Prague, Czech Republic

Background: Systemic sclerosis (SSc) is a multisystem, connective tissue disorder characterized by fibrosis of the skin and internal organ involvement, which can influence all aspects of life, including sexual life.

Objectives: This study aimed to compare sexual function in patients with SSc to age-/sex-matched healthy controls $(\mathrm{HC})$ and determine the potential impact of clinical features on sexual function.

Methods: In total, 90 women with SSc (mean age: 49.1, disease duration: 6.1 years, IcSSc/dcSSc: 62/28, mRSS: 9.3, ESSG activity index: 2.1), who fulfilled the ACR/ EULAR 2013 criteria, and 90 healthy controls (mean age: 49.1) without rheumatic diseases filled in 12 well-established and validated questionnaires assessing sexual function (FSFI, BISF-W, SFQ-28, SQoL-F), pelvic floor function (PISQ-12, PFIQ-7), fatigue (FIS, Fatigue Impact Scale), physical activity (HAP, Human Activity Profile), disability (HAQ, Health Assessment Questionnaire), depression (BDI-II, Beck's Depression Inventory-II) and quality of life (SF-36, Medical outcomes study Short Form 36 - PCS, Physical Component Summary; MCS, Mental Component Summary). A routine laboratory testing was performed. Data are presented as median (IQR).

Results: Patients with SSc reported significantly greater prevalence and severity of sexual dysfunction (FSFI, BISF-W, SFQ28 - in all subscales as well as total scores), worse sexual quality of life (SQLL-F) and pelvic floor dysfunction (PISQ-12, PFIQ-7) compared to HC (table 1). The prevalence of sexual dysfunction in patients with SSc according to the FSFI cut-off score was $77 \%$. Worse scores in SSc patients were associated with longer disease duration [BISF-W-total $(r=-0.243, p=0.026)$, FSFI-lubrication $(r=-0.229, p=0.035)]$, higher disease activity [ESSG activity index: BISF-W-total $(r=-0.291, p=0.010)$, FSFI-arousal $(r=-0.299, p=0.007)$ ], increased inflammation [CRP: BISF-W-receptivity/initiation $(r=-0.301, p=0.007)$ ], more pronounced fatigue [FIS-total: BISF-W-total $(r=-0.412, p<0.0001)]$, more severe depression [BDI-II: FSFI-total $(r=-0.506, p<0.0001)$, SQoL- $F(r=-0.369, p<0.0001)]$, worse functional disability [HAQ: FSFI-total $(r=-0.394, p<0.0001)]$, reduced physical activity [HAP: FSFI-total $(r=0.535, p<0.0001)$ ], and decreased overall quality of life [SF-36 PCS: FSFI-total $(r=0.428, p<0.0001)$, SF-36 MCS: SQoL-F $(r=0.472, p<0.0001)]$.

Table 1. Sexual function and pelvic floor function in women with SSc and healthy controls

\begin{tabular}{|c|c|c|c|}
\hline Questionnaire: score range (meaning) & $\operatorname{SSc}(n=90)$ & $\mathrm{HC}(\mathrm{n}=90)$ & p-value \\
\hline $\begin{array}{l}\text { FSFI: Female sexual function index: } 2 \\
\quad \text { (worst) - } 36 \text { (best) }\end{array}$ & $19.4(3.9-26.8)$ & $30.1(23.1-32.9)$ & $\mathrm{p}<0.0001$ \\
\hline $\begin{array}{l}\text { BISF-W: Brief Index of Sexual Function for } \\
\text { Women: -16 (worst) - } 75 \text { (best) }\end{array}$ & $14.3(2.1-35.1)$ & $38.2(19.3-46.2)$ & $p<0.0001$ \\
\hline $\begin{array}{l}\text { SQoL-F: Sexual Quality of Life Questionnaire- } \\
\text { Female: } 0 \text { (worst) - } 100 \text { (best) }\end{array}$ & -61.1 & $91.1(70.0-96.7)$ & $p<0.0001$ \\
\hline $\begin{array}{l}\text { PISQ-12: Pelvic Organ Prolapse/Urinary } \\
\text { Incontinence Sexual Questionnaire } \\
\text { short form: } 0 \text { (best) - } 48 \text { (worst) }\end{array}$ & $13.0(9.0-17.0)$ & $7.0(5.0-12.0)$ & $\mathrm{p}<0.0001$ \\
\hline $\begin{array}{l}\text { PFIQ-7: Pelvic Floor Distress Inventory } \\
\text { Questionnaire: } 0 \text { (best) - } 300 \text { (worst) }\end{array}$ & $9.5(0.0-48.8)$ & $0.0(0.0-8.3)$ & $\mathrm{p}<0.0001$ \\
\hline $\begin{array}{l}\text { SFQ-28: Sexual Functioning Questionnaire- } \\
28 \text { desire: } 5 \text { (worst) - } 31 \text { (best) }\end{array}$ & $17.0(12.0-20.0)$ & $21.0(17.0-23.0)$ & $\mathrm{p}<0.0001$ \\
\hline $\begin{array}{l}\text { SFQ-28 arousal sensation: } \\
4 \text { (worst) - } 20 \text { (best) }\end{array}$ & $10.0(8.0-13.0)$ & $12.0(9.0-15.0)$ & $p=0.0031$ \\
\hline SFQ-28 arousal lubrication: 2 (wo & $5.0(4.0-7.0)$ & 2-9.0) & $p<0.0001$ \\
\hline SFQ-28 arousal cognitive: 2 (worst) - 10 (best) & $5.0(4.0-6.0)$ & $7.0(5.0-8.0)$ & $p<0.0001$ \\
\hline SFQ-28 orgasm: 1 (worst) - 15 (best) & $10.0(6.5-12.0)$ & $12.0(10.0-13.0)$ & $p<0.0001$ \\
\hline SFQ-28 pain: 2 (worst) - 15 (best) & $12.0(9.5-15.0)$ & $15.0(13.0-15.0)$ & $p<0.0001$ \\
\hline SFQ-28 enjoyment: 6 (worst) - 30 (best) & $19.0(12.5-24.0)$ & $24.0(20.0-25.8)$ & $p<0.0001$ \\
\hline SFQ-28 partner: 2 (worst) - 10 (best) & $9.0(8.0-10.0)$ & 10. $(9.0-10.0)$ & $p=0.0182$ \\
\hline
\end{tabular}

Conclusion: Women with SSc reported significantly impaired sexual function and pelvic floor function compared to age-/sex-matched healthy controls. Worse scores in SSc were associated with disease-related features.

Acknowledgements: Supported by MHCR 023728, GA UK 1578119, and SVV 260373.

Disclosure of Interests: None declared

DOI: 10.1136/annrheumdis-2021-eular.1829

\section{POS0847 IDENTIFICATION OF MOLECULAR PHENOTYPES IN SYSTEMIC SCLEROSIS BY INTEGRATIVE SYSTEMS ANALYSIS}

M. J. Chang ${ }^{1,2,3}$, S. X. Zhang ${ }^{1,2,3}$, Q. Wang ${ }^{4,5}$, J. Qiao ${ }^{1,2,3}$, R. Zhao ${ }^{1,2,3}$, S. Song ${ }^{1,2,3}$, Y. Zhang ${ }^{1,2,3}$, Q. Yu ${ }^{6}$, P. F. He ${ }^{5}$, X. Li ${ }^{1,2,3}$. ${ }^{1}$ The Second Hospital of Shanxi Medical University, Department of Rheumatology, Taiyuan, China; ${ }^{2}$ Shanxi Li Xiaofeng Medical Groups, Department of Rheumatology, Taiyuan, China; ${ }^{3}$ Ministry of Education, Key Laboratory of Cellular Physiology at Shanx Medical University, Taiyuan, China; ${ }^{4}$ Shanxi Medical University, Basic Medical College, Taiyuan, China; ${ }^{5}$ Shanxi Medical University, Medical Data Sciences, Taiyuan, China; ${ }^{6}$ Shanxi Medical University, Medical Data Sciences/School of Management, Taiyuan, China

Background: Systemic sclerosis (scleroderma, SSc) is a systemic autoimmune disease characterized by inflammation, fibrosis and vasculopathy and associated with high mortality and high morbidity ${ }^{1}$. Stratification based on whole-genome gene expression data could provide a new basis for clinical diagnosis from a micro perspective ${ }^{2}$.

Objectives: The objective of this study is to stratify patients with SSc, combine with clinical skin scores and clinical features, and provide a preliminary assess ment and novel insights for assessing disease severity, and treatment design. Methods: The original data mRNA expression profiles of GSE95065 (including 18 SSc patients and 4 healthy controls) and GSE130955 (including 58 SSc patients and 33 healthy controls) were downloaded from the public Gene Expression Omnibus (GEO) database. After batch correction, background adjustment, and other pre-processing, a large gene matrix was obtained to identify the dif ferently expressed genes (DEGs) of SSc compared with healthy controls. Then the gene expression matrix decomposition was used to identify SSc subtypes by NMF algorithm. The cluster-based signature genes were applied to pathway enrichment analysis by Metascape ${ }^{3}$. Immune infiltrating cells and clinical skin scores were evaluated in all SSc subtypes.

Results: Total 325 DEGs were imputed to NMF unsupervised machine learning algorithm. Patients were divided into 2 subtypes (Figure 1A), one of which (sub1) was mostly enriched in the defense response to bacterium and cellular response to lipopolysaccharide pathway and another subtype (sub2) was enriched in the PPAR signaling and alcohol metabolic process pathway (Figure 1B-C). According to immune infiltration, sub1 had higher level of immune cells such as $B$ cells CD4+T cells, DC cells, Th2 cells and Tregs compared with sub2 $(P<0.01)$. Sub2 had more skin-related cells, including Epithelial cells, Fibroblasts and Sebocytes $(P<0.05)$. Interestingly, combined with clinical information, sub1 showed a severe clinical skin score over those of Sub2 patients $(P<0.05)$ (Figure 1D-E) Conclusion: Our findings indicated that SSc patients could be stratified into 2 subtypes which had different molecular profiles of disease progression and
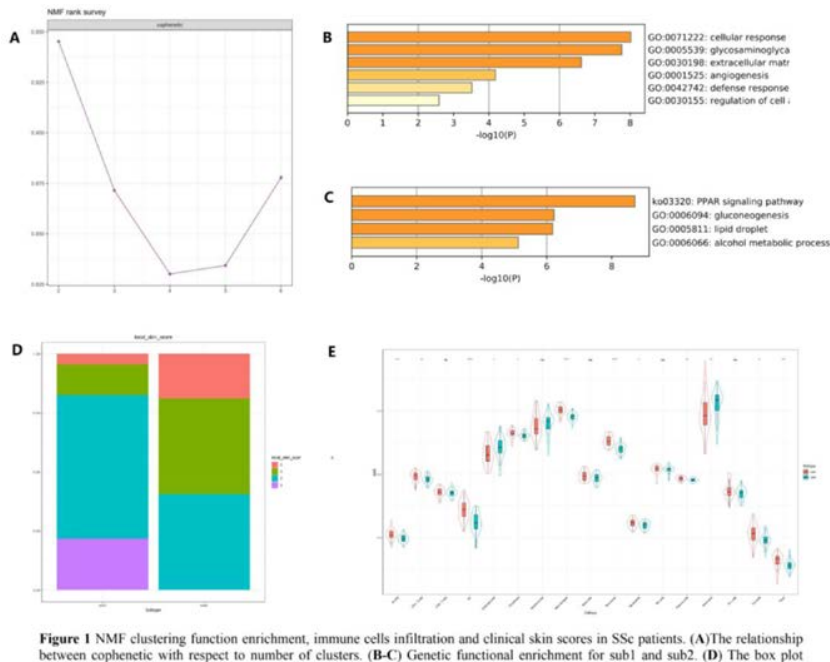

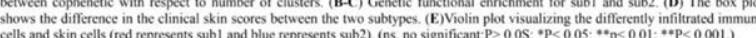

